\title{
LA ADMINISTRACIÓN DE EFECTIVO Y EL CONCEPTO DE FLOTACIÓN
}

\author{
CASH MANAGEMENT AND THE CONCEPT OF FLOATING \\ Félix Hipólito Pajuelo Chauca* \\ Docente Auxiliar de la Facultad de Ciencias Contables \\ Universidad Nacional Mayor de San Marcos - UNMSM / Lima - Perú \\ [Recepción: Setiembre de 2015 / Conformidad: Octubre 2015]
}

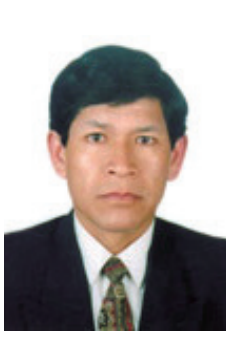

\section{RESUMEN}

El presente artículo, tuvo como propósito indagar algunas situaciones concretas en relación a la optimización del manejo de efectivo y contribuir con los responsables del área de tesorería de las empresas en la administración eficaz y eficiente del efectivo; con el único objetivo de lograr o generar un flujo adecuado de dinero líquido que permita a la compañía cumplir con el pago de sus obligaciones operativas y en general, lograr la rentabilidad del dinero a satisfacción de los accionistas.

En tal sentido, la flotación neta (o simplemente flotación) que es la diferencia monetaria entre el saldo bancario de la empresa y su saldo de efectivo en libros; generado como consecuencia de la demora en la transferencia de los cheques vía correo (demora postal), sean procesados por la empresa receptora (demora por procesamiento) y sean compensados a través del sistema bancario (demora por compensación); es una situación concreta en términos de días, en el que el responsable del área de tesorería de la empresa podría generar grandes ahorros (uso de la flotación), $\mathrm{y}$ adquirir valores negociables por ejemplo y tener una rentabilidad considerable. Siempre que aplique medidas drásticas para acelerar el proceso de los cheques en cobranza; y a la vez, que demore tanto tiempo como sea posible en el pago de los cheques emitidos.

\section{Palabras clave:}

Administración eficaz; desembolsos en tránsito; cobranzas en tránsito; uso de flotación; disponible; rentabilidad.

\begin{abstract}
This article aims to investigate some specific situations related to the optimization of cash management and to contribute with officials from the Treasury of companies in the effective and efficient management of cash; with the sole aim of achieving or generating an adequate cash flow that allows the company to meet its operational payment obligations and generally achieving money profitability to satisfy shareholders. In this regard, the floating net (or simply floating) -which is the monetary difference between the bank balance of the company and its cash balance booksis generated as a result of the delay in the transfer of checks via mail (postal delay) to be processed by the receiving company (processing delay) and could be compensated through the banking system (delay compensation). This is a particular situation in terms of days in which the Head of treasury of the company could generate large savings (use of flotation), and acquire such securities and have considerable profitability. Whenever you apply drastic measures to accelerate the process of collecting checks, as well as delayed as long as possible in the payment of checks issued.
\end{abstract}

\section{Keywords:}

Effective management; disbursements in transit; collections in transit; Use of flotation; available; cost effectiveness.

* Contador Público Colegiado. Email: fpajuelo105@hotmail.com 


\section{INTRODUCCIÓN}

La generación de efectivo es quizás uno de los principales objetivos que tienen los ejecutivos responsables en las diferentes empresas; sean estas, pequeñas, medianas o grandes empresas. La mayoría de sus actividades y/o acciones están encaminadas a generar de manera directa e indirecta un flujo apropiado de dinero líquido que, entre otros aspectos, pueda financiar sus actividades operativas o puedan ser invertidas para sostener el crecimiento del negocio; así como, efectuar el pago de sus pasivos a corto plazo a su vencimiento y en general, a retribuir a los accionistas con una rentabilidad que supere sus expectativas.

La actividad de la tesorería en las finanzas de las empresas tiene entre sus objetivos invertir los excedentes generados en activos financieros; así como, la búsqueda de formas de endeudamiento menos costosas. Es una parte esencial de la gestión de tesorería, lograr un adecuado equilibrio entre los beneficios y los costos de liquidez, función que realiza el tesorero al decidir sobre la distribución de los activos líquidos, entre efectivo y valores negociables.

En términos más concretos, un negocio es rentable, solo cuando genera una cantidad relativamente suficiente de dinero y que supere sus egresos correspondientes.

La administración de efectivo casi siempre se centraliza alrededor de dos áreas: el de presupuesto de efectivo y el control interno de contabilidad. El presupuesto de caja o presupuesto de efectivo es una herramienta de gestión que prevé las futuras entradas y salidas de efectivo, permitiendo que la empresa programe sus necesidades de corto plazo; por lo que el área financiera debe prestar especial atención a la planeación de sus excedentes o déficits, ya que al obtener remanentes estos pueden ser invertidos, pero por el contrario, si hay faltante planear la forma de buscar financiamiento a corto plazo.

Por otro lado, el control contable es necesario para dar un sustento a la planeación y además, con el fin de asegurar que el efectivo se utilice de manera correcta en operaciones propias del negocio y no para otros fines. Se necesita un sistema de control interno adecuado para prevenir robos y evitar que los empleados utilicen el dinero de la empresa para fines personales. El control interno no se diseña para detectar errores, sino para reducir la oportunidad que ocurran los malos manejos.

\section{MARCO CONCEPTUAL}

\section{Efectivo}

Según Contadores \& Empresas (2012), es el efectivo mantenido en caja y depósitos a la vista.

En el Diccionario de Contabilidad y Finanzas (2002), el efectivo se define como el dinero líquido en poder de una empresa, particular, etc. o en depósito en las instituciones financieras.

Por efectivo entendemos que es el activo financiero, el dinero contante y sonante que se tiene en caja (billetes y monedas), constituyen dinero en efectivo los que se tiene en los diferentes bancos depositados en la modalidad de depósitos a la vista (tratándose de empresas). Por el lado de las personas, es el dinero que se tiene en el bolsillo o el dinero depositado en el banco en una cuenta corriente.

También podemos definir el efectivo como los fondos que mantienen las empresas y que están disponibles para los desembolsos inmediatos. 


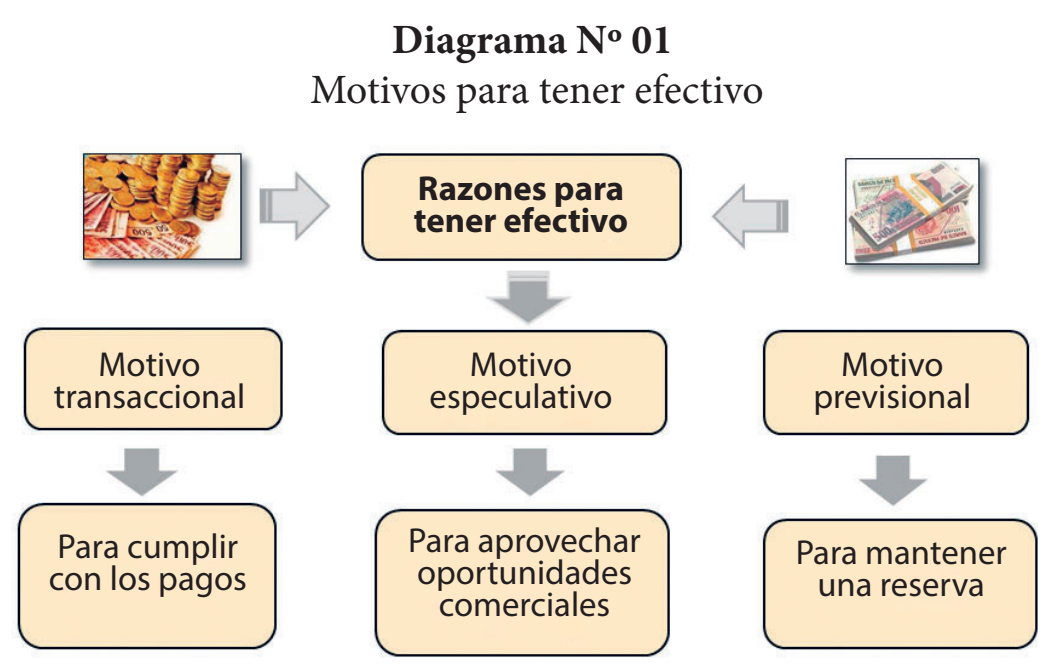

Fuente: Elaboración propia.

\section{Flotación}

Según Besley y Brigham (2001), la flotación es la diferencia entre el saldo que se muestra en la cuenta de cheques de una empresa (o un individuo) y el saldo en los registros del banco (p. 641).

Van Horne y Wachowicz (2002), señalaban a la flotación como la diferencia entre el saldo en la cuenta de cheques de una empresa (o persona) y el saldo en los libros del banco (p. 232).

Para Weston (1994), define la flotación comola diferencia que existe entre el saldo que aparece en la chequera de una empresa (o un individuo) y el saldo que arrojan los registros bancarios (p. 489).

A partir de las definiciones podemos establecer que, la flotación no es otra cosa que la diferencia entre el saldo disponible o el saldo en el Banco según extracto o reporte bancario y el saldo contable o saldo en el libro bancos o también llamado, saldo del Libro Mayor. En consecuencia, si se quiere establecer o determinar el monto de las partidas en tránsito (flotación) se puede aplicar esta sencilla ecuación:

\section{Flotación = Saldo Disponible - Saldo Contable}

\section{Compensación de cheques}

Es el proceso de conversión en efectivo de un cheque que ha sido emitido y enviado por correo y que se deposita en la cuenta del pagador. Besley y Brigham (2001, 641).
Según Weston (1994), compensación de un cheque es el proceso que se sigue para convertir en efectivo y depositar a la cuenta del beneficiario un cheque que ha sido emitido y enviado por correo (p. 489).

$\mathrm{Al}$ respecto, podemos comentar que cuando un cliente emite y envía por correo un cheque, no necesariamente los fondos estarán disponibles de manera inmediata para la empresa que recibe. En alguna ocasión, por ejemplo hemos depositado un cheque en nuestra cuenta corriente y posteriormente, nos hemos dado con la sorpresa de que aún no se puede girar cheques contra este depósito hasta que el proceso de compensación del cheque haya terminado. Esto significa que el banco debe primero asegurarse, que el cheque que depositamos no tenga ningún error o falla y recibir posteriormente, los fondos del banco del cliente y facilitarnos el efectivo correspondiente.

Tal como señalaba Weston, primeramente un cheque debe ser entregado a través del correo y posteriormente, debe ser compensado a través del sistema bancario antes de que el dinero se pueda usar.

Esto significa por ejemplo, que los cheques que reciben las empresas de sus clientes ubicados en ciudades distantes están especialmente sujetos a las demoras debido al tiempo que demoran los envíos postales y a la vez, también porque hay más partes involucradas. A manera de otro ejemplo, supongamos que recibimos un cheque y que lo depositamos en nuestro banco. Este debe enviar el cheque al banco 
contra el cual fue girado. Solo después de que este último banco haya transferido los fondos a nuestro banco, los fondos estarán disponibles para que lo podamos retirar. Así, el plazo de tiempo que se requiere para la compensación de los cheques es una función de la distancia que media entre el banco del pagador y el banco del beneficiario.

\section{Desembolsos en tránsito}

Stephen (2010), señalaba que los cheques girados por una empresa son las que generan los desembolsos en tránsito, lo que ocasiona una disminución en el saldo en libros de la empresa, aunque ningún cambio en el saldo disponible. Asimismo, mencionaba que los cheques recibidos por la empresa generan cobranza en tránsito. Estos fondos incrementan los saldos en libros, pero no modifican de inmediato los saldos disponibles (p. 614).

Esto a la luz de las operaciones que realizan las empresas, significa que el giro de un cheque constituye la salida de efectivo (desembolsos en tránsito), por lo que disminuye el saldo en libros de la empresa. Sin embargo, no genera ningún cambio en el saldo disponible. En tanto que los cheques recibidos por la compañía crean cobranzas en tránsito (ingreso de efectivo), aumentando los saldos en libros, sin modificar de inmediato los saldos disponibles con que cuenta la empresa.

\section{EXPLICACIÓN DE LA FLOTACIÓN (Partidas en tránsito)}

Desde el punto de vista de la Persona Natural, cuando uno expide un cheque a nombre de un titular, existe una demora antes de que los fondos puedan ser retirados por el titular de su cuenta corriente en bancos. Esta demora se debe a aspectos tales como el tiempo que necesita el banco para procesar el cheque y el tiempo que transcurre antes de que el sistema bancario retire los fondos de su cuenta corriente. $\mathrm{Du}$ rante este lapso de tiempo, uno se está beneficiando con la flotación.

Similar situación ocurre en el caso de las Personas Jurídicas, el saldo de efectivo que una empresa refleja en sus libros de llama Saldo en Libros o saldo del Libro Mayor. El saldo que figura en los saldos del reporte bancario y del cual se puede disponer se llama saldo disponible o saldo abonado en cuenta corriente. A la diferencia entre el saldo disponible y el que aparece en el libro mayor se le denomina partidas en tránsito y representa el efecto neto de los cheques en proceso de compensación (movimiento que siguen los cheques en el sistema bancario).

\section{APLICACIÓN PRÁCTICA}

Caso $\mathbf{N}^{\circ} 01$ : La compañía NBC emite cheques cada dos semanas por un promedio de $\$ 200,000$, que requieren de tres días para ser compensados. ¿Qué cantidad de efectivo podrá ahorrar anualmente durante estos tres días de demora, la transferencia de fondos de una cuenta que genera intereses de un $0.05 \%$ por día?

Solución: Para darle solución al caso planteado aplicamos la siguiente ecuación simple:

\section{Flotación = Saldo Disponible - Saldo Contable}

En donde la flotación es la diferencia entre el saldo disponible o saldo en el banco y el saldo contable o saldo del libro mayor de la empresa. 
Cuadro No 01

Caso Flotación de Efectivo

\begin{tabular}{|c|c|c|c|c|c|c|c|}
\hline Meses & $\begin{array}{c}\text { Saldo } \\
\text { Disponible }\end{array}$ & $\begin{array}{c}\text { Emisión de } \\
\text { Cheques }\end{array}$ & $\begin{array}{c}\text { Saldo en } \\
\text { Libro Banco }\end{array}$ & $\begin{array}{l}\text { Partida en } \\
\text { Transito }\end{array}$ & $\begin{array}{l}\text { \# Días demora } \\
\text { Compensación }\end{array}$ & $\begin{array}{l}\text { T. Interes } \\
\text { diaria }\end{array}$ & $\begin{array}{l}\text { Total de } \\
\text { Ahorros }\end{array}$ \\
\hline Diciembre & 200,000 & 200,000 & - & 200,000 & 3 & $0.05 \%$ & 300.00 \\
\hline Enero & 200,000 & 200,000 & - & 200,000 & 6 & $0.05 \%$ & 600.00 \\
\hline Febrero & 200,000 & 200,000 & - & 200,000 & 6 & $0.05 \%$ & 600.00 \\
\hline Marzo & 200,000 & 200,000 & - & 200,000 & 6 & $0.05 \%$ & 600.00 \\
\hline Abril & 200,000 & 200,000 & - & 200,000 & 6 & $0.05 \%$ & 600.00 \\
\hline Mayo & 200,000 & 200,000 & - & 200,000 & 6 & $0.05 \%$ & 600.00 \\
\hline Junio & 200,000 & 200,000 & - & 200,000 & 6 & $0.05 \%$ & 600.00 \\
\hline Julio & 200,000 & 200,000 & - & 200,000 & 6 & $0.05 \%$ & 600.00 \\
\hline Agosto & 200,000 & 200,000 & - & 200,000 & 6 & $0.05 \%$ & 600.00 \\
\hline Setiembre & 200,000 & 200,000 & - & 200,000 & 6 & $0.05 \%$ & 600.00 \\
\hline Octubre & 200,000 & 200,000 & - & 200,000 & 6 & $0.05 \%$ & 600.00 \\
\hline Noviembre & 200,000 & 200,000 & - & 200,000 & 6 & $0.05 \%$ & 600.00 \\
\hline Diciembre & 200,000 & 200,000 & - & 200,000 & 6 & $0.05 \%$ & 600.00 \\
\hline Enero & 200,000 & 200,000 & - & 200,000 & 3 & $0.05 \%$ & 300.00 \\
\hline TOTAL & & $2,800,000$ & & $2,800,000$ & 78 & & $7,800.00$ \\
\hline
\end{tabular}

Fuente: Elaboración propia.

\section{Comentario:}

Elvalor de los cheques que ha emitido la empresa pero que aún, están siendo procesados y que por lo tanto, no han sido deducidos por el banco del saldo de la cuenta de la empresa, es lo que constituye la flotación.

En el caso planteado, la compañía podrá ahorrar un monto anual de $\$ 7,800$ por concepto de flotación o demora en la transferencia de efectivo, que bien lo podría invertir en la adquisición de valores negociables a corto plazo o aprovechar otras formas de rentabilizar el efectivo.

Caso $\mathbf{N}^{\circ}$ 02: Supongamos que la empresa MGS tiene en la actualidad 10,000 dólares depositados en el banco. El 10 de junio compra materias primas y paga con cheque por 10,000 dólares, el proveedor lo cobra el 16 de junio. Por otro lado, consideramos que el 10 de noviembre MGS recibe un cheque de un cliente por 10,000 dólares. Asimismo, la empresa tiene depositado en el banco 10,000 dólares y cero partidas en tránsito.

Solución: En el caso planteado, se procede a establecer los desembolsos en tránsito; así como las cobranzas en tránsito, recurriendo a unas ecuaciones simples:
Partidas en tránsito $=$ Saldo disponible de la empresa - Saldo en libros de la empresa

$$
\begin{aligned}
& =\$ 10,000 \text { dólares }-10,000 \\
& =0 \text { dólares }
\end{aligned}
$$

La situación de MGS del 10 al 16 de junio será:

Desembolso en tránsito $=$ Saldo disponible de la empresa - Saldo en libros de la empresa

$$
\begin{aligned}
& =\$ 10,000 \text { dólares }-0 \\
& =10,000 \text { dólares }
\end{aligned}
$$

En el lapso de tiempo que tarda la compensación de cheque, MGS tiene un saldo en el banco de $\$ 10,000$ dólares, pudiendo beneficiarse de este dinero durante este periodo. Por ejemplo con el saldo disponible podría adquirirse temporalmente valores negociables y ganar un interés.

Partidas en tránsito $=$ Saldo disponible de la empresa - Saldo en libros de la empresa

$$
\begin{aligned}
& =\$ 10,000 \text { dólares }-10,000 \\
& =0 \text { dólares }
\end{aligned}
$$

La situación de MGS del 10 al 12 de noviembre será: Cobranza en tránsito = Saldo disponible de la empresa - Saldo en libros de la empresa

$$
\begin{aligned}
& =\$ 10,000 \text { dólares }-20,000 \\
& =-10,000 \text { dólares }
\end{aligned}
$$


A manera de comentario, las actividades de pago de las empresas son las que generan desembolsos en tránsito y las actividades de cobranza generan cobranza en tránsito. El efecto neto; es decir, la suma de los desembolsos y las cobranzas en tránsito totales, constituyen las partidas netas en tránsito.

Las empresas debieran mostrar más interés por las partidas netas en tránsito y el saldo disponible que el saldo en libros. Si el administrador financiero tiene información de que un cheque girado por la empresa no será compensado hasta después de varios días, podría mantener un saldo menor de efectivo en el banco. Esto podría generar muchísimo dinero para el negocio.

\section{CONCLUSIONES}

1. La flotación es la diferencia entre el saldo disponible y el saldo contable.

2. Las demoras que ocasiona la flotación surgen porque se requiere de un determinado tiempo para que los cheques sean transferidos a través del correo (demora postal), sean procesados por la empresa receptora (demora por procesamiento) y sean compensados por el sistema bancario (demora por compensación).

3. La magnitud de la flotación neta de una empresa es una función de su capacidad para acelerar las cobranzas de los cheques recibidos y retardar el pago de los cheques emitidos.
4. Las empresas para ser eficientes en la administración del efectivo, deben tomar medidas bastante drásticas para acelerar el procesamiento de los cheques que reciben, con el propósito de poner a trabajar los fondos con mayor rapidez, a la vez, que deben tratar de demorar sus propios pagos el tiempo que sea posible.

5. El valor del dinero en el tiempo es un principio fundamental de las finanzas.

\section{REFERENCIAS BIBLIOGRÁFICAS}

1. BESLEY, S. \& BRIGHAM, E. (2001) Fundamentos de administración financiera. 12a Edicion. Mc Graw-Hill Interamericana de Editores S.A.

2. STEPHEN, R. (2010) Fundamentos de finanzas corporativas, Novena edición.

3. CONTADORES \& EMPRESAS (2012) Diccionario Aplicativo para Contadores.

4. WESTON, B. (1994) Fundamentos de administración financiera; Interamericana de México, S.A. McGraw-Hill. Décima edición.

5. VAN HORNE, J.C. y WACHOWICZ (2002) Fundamentos de administración financiera, Edición Pearson Educación.

6. Diccionario de Contabilidad y Finanzas Cultural, S.A. Edición 2002. 